\title{
Defining genetic risk factors for scleroderma-associated interstitial lung disease
}

\author{
IRF5 and STAT4 gene variants are associated with scleroderma while STAT4 is protective against \\ scleroderma-associated interstitial lung disease
}

\author{
Carmel J. W. Stock ${ }^{1}$ (1) - Angelo De Lauretis ${ }^{1,2} \cdot$ Dina Visca $^{1,3} \cdot$ Cecile Daccord $^{1,4} \cdot$ Maria Kokosi $^{1} \cdot$ Vasilis Kouranos $^{1}$. \\ George Margaritopoulos ${ }^{1}$. Peter M. George ${ }^{1}$. Philip L. Molyneaux ${ }^{1}$ - Svetlana Nihtyanova ${ }^{5}$. Felix Chua ${ }^{1}$. \\ Toby M. Maher ${ }^{1}$. Voon Ong ${ }^{5}$ - David J. Abraham ${ }^{5}$. Christopher P. Denton ${ }^{5} \cdot$ Athol U. Wells $^{1} \cdot$ Louise V. Wain $^{6,7}$. \\ Elisabetta A. Renzoni ${ }^{1}$
}

Received: 26 September 2019 / Revised: 19 December 2019 / Accepted: 29 December 2019 / Published online: 8 January 2020

(C) The Author(s) 2020

\begin{abstract}
Although several genetic associations with scleroderma (SSc) are defined, very little is known on genetic susceptibility to SSc-associated interstitial lung disease (SSc-ILD). A number of common polymorphisms have been associated with SScILD, but most have not been replicated in separate populations. Four SNPs in IRF5, and one in each of STAT4, CD226 and $I R A K 1$, selected as having been previously the most consistently associated with SSc-ILD, were genotyped in 612 SSc patients, of European descent, of whom 394 had ILD. The control population $(n=503)$ comprised individuals of European descent from the 1000 Genomes Project. After Bonferroni correction, two of the IRF5 SNPs, rs 2004640 (OR (95\% CI) 1.30 $\left.(1.10-1.54), p^{\text {corr }}=0.015\right)$ and $\mathrm{rs} 10488631\left(\right.$ OR $\left.1.48(1.14-1.92), p^{\text {corr }}=0.022\right)$, and the STAT4 SNP rs7574865 (OR 1.43 $\left.(1.18-1.73), p^{\text {corr }}=0.0015\right)$ were significantly associated with SSc compared with controls. However, none of the SNPs were significantly different between patients with SSc-ILD and controls. Two SNPs in IRF5, rs10488631 (OR 1.72 (1.24$\left.2.39), p^{\text {corr }}=0.0098\right)$, and rs2004640 (OR $\left.1.39(1.11-1.75), p^{\text {corr }}=0.03\right)$, showed a significant difference in allele frequency between controls and patients without ILD, as did STAT4 rs7574865 (OR 1.86 $(1.45-2.38), p^{\text {corr }}=6.6 \times 10^{-6}$ ). A significant difference between SSc with and without ILD was only observed for STAT4 rs 7574865 , being less frequent in
\end{abstract}

Previous publications The data in this manuscript has been presented at the European Respiratory Society Congress 2019 and at the British Thoracic Society Winter 2019 meeting, conference proceedings not yet published.

Electronic supplementary material The online version of this article (https://doi.org/10.1007/s10067-019-04922-6) contains supplementary material, which is available to authorized users.

Carmel J. W. Stock

c.stock@imperial.ac.uk

1 Interstitial Lung Disease Unit, National Heart and Lung Institute, Imperial College London, Royal Brompton and Harefield NHS Foundation Trust, Sydney Street, London SW3 6NP, UK

2 Unita' Operativa Malattie Respiratorie, Ospedale Guido Salvini, Garbagnate Milanese, Milan, Italy

3 Division of Pulmonary Rehabilitation, Istituti Clinici Scientifici Maugeri, IRCCS, Tradate, Italy
4 Division of Respiratory Medicine, Lausanne University Hospital (CHUV), Lausanne, Switzerland

5 Centre for Rheumatology and Connective Tissue Diseases, Royal Free and University College Medical School, London, UK

6 Department of Health Sciences, University of Leicester, Leicester, UK

7 National Institute for Health Research, Leicester Respiratory Biomedical Research Centre, Glenfield Hospital, Leicester, UK 
patients with ILD (OR $\left.0.66(0.51-0.85), p^{\text {corr }}=0.0084\right)$. In conclusion, IRF5 rs2004640 and rs10488631, and STAT4 rs7574865 were significantly associated with SSc as a whole. Only STAT4 rs7574865 showed a significant difference in allele frequency in SSc-ILD, with the T allele being protective against ILD.

Key points

- We confirm the associations of the IRF5 SNPs rs2004640 and rs10488631, and the STAT4 SNP rs7574865, with SSc as a whole.

- None of the tested SNPs were risk factors for SSc-ILD specifically.

- The STAT4 rs 7574865 Tallele was protective against the development of lung fibrosis in SSc patients.

- Further work is required to understand the genetic basis of lung fibrosis in association with scleroderma.

Keywords CD226 · Genetic association · Genetics $\cdot$ IRAK1 $\cdot$ IRF5 $\cdot$ SSc-ILD $\cdot$ STAT4

\section{Introduction}

Scleroderma ( $\mathrm{SSc}$ ) is a chronic connective tissue disease characterised by fibrosis of the skin and internal organs, vascular damage and immune dysregulation [1]. SSc is characterised by marked heterogeneity of clinical manifestations and disease course, and its pathogenesis remains poorly understood. SSc carries one of the highest mortality rates among connective tissue diseases, with interstitial lung disease (ILD) being the leading cause of death [2]. Although the majority of SSc-ILD patients have a relatively mild and/or stable lung disease, a substantial minority have progressive lung fibrosis [2]. The molecular pathways which underlie development and progression of SSc-ILD are currently unknown, but are likely to be driven by an interaction between predisposing genetic factors and environmental triggers. Identification of the genetic determinants of lung fibrosis in SSc could improve understanding of pivotal molecular pathways, potentially leading to better prognostic and therapeutic tools for SSc-ILD.

Evidence for a genetic predisposition to SSc as a whole includes a higher prevalence in first degree relatives, and variation in prevalence among different ethnic groups. Twin studies have revealed a strong genetic influence on antinuclear antibody status, in turn linked with internal organ involvement, with ATA antibodies strongly associated with development of SSc-ILD, and ACA antibodies protective for ILD. A number of genes have been consistently associated with SSc as a whole. Similarly to other autoimmune diseases, there is a strong effect of the HLA (human leukocyte antigen) region, mainly with specific autoantibodies [3]. Immune responserelated genes are among the most consistently replicated non-HLA associations, including interferon regulatory factor 5 (IRF5) $[4,5]$, signal transducer and activator of transcription 4 (STAT4) [6, 7], and cell receptor CD3 (CD247) [8].

A smaller number of studies have looked at SSc-ILD specifically, although conflicting evidence is reported, with only a few associations replicated in more than one study [9]. Genetic associations reported as specific to SSc-ILD in more than one cohort, including IRF5, STAT4, DNAX accessory molecule 1 (CD226) and interleukin-1 receptor-associated kinase-1 (IRAK1). A number of SNPs in IRF5 have been associated with SSc-ILD, including in a French $[10,11]$ and a Han Chinese [12] population. IRF5 SNP rs4728142 was associated with improved survival in SSc [4]. A SNP in STAT4, rs7574865, has also been associated with SSc-ILD in a French [13] and a Han Chinese [7] population. CD226 SNP rs763361 was significantly associated with SSc-ILD in a meta-analysis study of three European populations, with a trend towards significance when each population was analysed separately [14]. The minor allele of rs 1059702 in IRAKI, on the $\mathrm{X}$ chromosome, results in increased NFK-B activity. Two studies performing meta-analysis on European populations have been reported. In both studies, which both comprised meta-analysis of three populations, rs1059702 was associated with SSc-ILD $[15,16]$.

In this study, we focused on genes previously reported as risk factors for SSc-ILD in more than one population, and selected the SNPs which had been most consistently associated [9]. We also sought to determine their association with mortality and ILD progression.

\section{Materials and methods}

\section{Study populations}

DNA samples were collected from consecutive, unrelated SSc patients attending clinics at the Royal Brompton and Royal Free Hospitals, London. The diagnoses were made from welldefined criteria for SSc [17]. Only individuals of European descent were included. The control population $(n=503)$ comprised individuals of European descent from the publicly available 1000 Genomes Project [18].

\section{Clinical assessment}

ILD was defined as the presence of fibrosis on chest imaging (chest X-ray or HRCT) and/or a forced vital capacity (FVC) < $75 \%$. Pulmonary function tests (expressed as percent predicted) from the time of first presentation at the Royal Brompton Hospital were available for 578 patients. As a marker of ILD severity which adjusts for the extent of emphysema, the composite physiological index (CPI) was calculated as $\mathrm{CPI}=91.0$ 
$-(0.65 \times \mathrm{DLCO} \%$ predicted $)-(0.53 \times \mathrm{FVC} \%$ predicted $)+$ $(0.34 \times$ FEV1\% predicted) [19]. Time to decline was quantified using serial pulmonary functional indices starting from first visit. Significant functional deterioration was defined as a decline (quantified as percentage change from baseline) of $\geq$ $10 \%$ in FVC and/or of $\geq 15 \%$ in DLCO. To allow for possible response to treatment or spontaneous fluctuations, time to irreversible decline was used, defined as time to first significant change observed on at least two consecutive occasions. Data at a sufficient number of time points was available to calculate time to decline in 374 patients. All-cause mortality was also analysed $(n=553)$.

\section{Genotyping}

The gene locations of the seven SNPs selected for testing are shown in Fig. 1. DNA was extracted from blood using Gentra PureGene DNA kits (Qiagen). Genotyping was carried out according to manufacturer's instructions using a commercially available TaqMan ${ }^{\circledR}$ assay and TaqMan $®$ universal PCR master mix, no AmpErase® UNG (Applied Biosystems), on a Rotor-Gene 6000 real-time PCR machine (Qiagen). Quality control and genotype determination were performed using the Rotor Gene 6000 Series Software 1.7 (Corbett Research).

\section{Statistical analysis}

To test for deviation from Hardy-Weinberg equilibrium (HWE), genotype frequencies were determined by direct counting, and the chi square statistic or Fisher's exact test were used as appropriate. Chi square analyses for association were carried out in Unphased v 3.1. To assess the most appropriate genetic model for significant signals, logistic regression analysis was applied in STATA v 15 . Only female patients $(n=483)$ and controls $(n=$ 263 ) were included in the analysis of the $\mathrm{X}$ chromosome IRAKI SNP. Bonferroni correction was applied to correct for multiple testing of seven SNPs. A corrected $p$ value $\left(p^{\text {corr }}\right)<0.05$ was considered significant. The current study had $80 \%$ power to detect an association with SSc as a whole with an OR of at least 1.5 for a SNP with a minor allele frequency of 0.5 at $p^{\text {corr }}<0.05$, and
$80 \%$ power to detect an association with SSc-ILD with an OR of at least 1.6. Cox proportional hazards analysis was used to evaluate time to decline in FVC, time to decline in DLCO, and mortality, as implemented in the Stata v 15.1 (Computing Resource Centre).

\section{Results}

A total of 612 patients were included in the study, of whom 394 had ILD. Patient demographic and clinical characteristics are shown in Table 1. The genotyping success rate for all seven SNPs was $\geq 97.7 \%$. All seven SNPs conformed to Hardy-Weinberg equilibrium in the control population.

As shown in Table 2, a total of three of the tested SNPs were significantly associated with SSc compared with controls. IRF5 rs2004640 T allele (OR $1.30(95 \%$ CI $\left.1.10-1.54), p^{\text {corr }}=0.015\right)$, IRF5 rs10488631 C allele (OR 1.48 (95\% CI 1.14-1.92), $p^{\text {corr }}=$ 0.022 ) and STAT4 rs7574865 T allele (OR 1.43 (95\% CI 1.18$\left.1.73), p^{\text {corr }}=0.0015\right)$ were risk factors for SSc.

No significant difference in allele frequency of the tested SNPs was observed between patients with SSc-ILD and controls (Table 2). However, the minor allele of two SNPs in IRF5 were more frequent in SSc patients without ILD than in controls, rs2004640 T allele (OR 1.39 (95\% CI 1.11-1.75), $p^{\text {corr }}=$ 0.03 ) and rs10488631 C allele (OR 1.72 (95\% C 1.24-2.39), $\left.p^{\text {corr }}=0.0098\right)$. STAT4 SNP rs7574865 T allele was also significantly associated with SSc-non ILD (OR 1.86 (95\% CI $\left.1.45-2.38), p^{\text {corr }}=6.6 \times 10^{-6}\right)$. STAT4 rs7574865 T allele was significantly less frequent in SSc patients with ILD than those without (OR $0.66(95 \%$ CI $\left.0.51-0.85), p^{\text {corr }}=0.0084\right)$ (Table 2). A logistic regression analysis using an additive model provided comparable results (Supplementary table 1).

Given the higher proportion of females in the patient cohort, $80.9 \%$ compared with $52.3 \%$ in the control population, we performed a logistic regression with sex as a covariate and observed no change to the significance of the association with any of the variants (Supplementary table 2).

None of the seven tested SNPs were associated with mortality (Table 3). An association was seen between IRF5

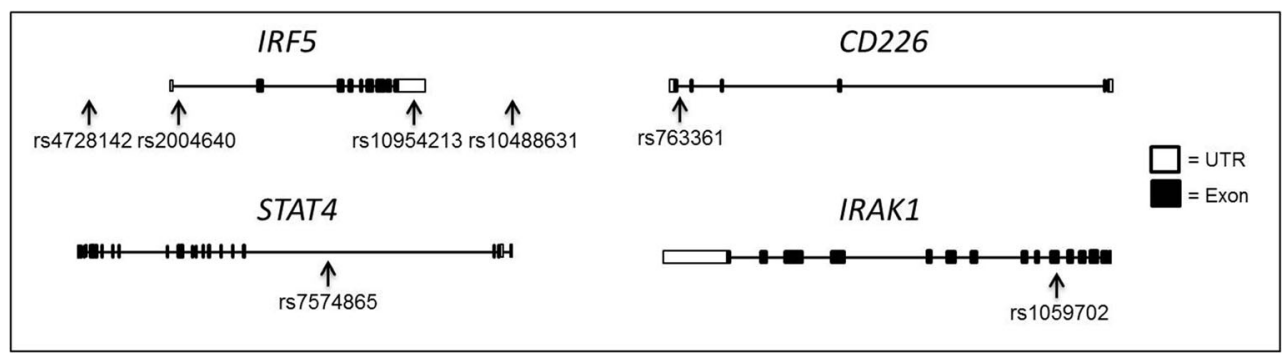

Fig. 1 Location of the studied SNPs in IRF5, CD226, STAT4 and IRAK1. Shown are the locations of the SNPs tested in this study in relation to the gene exons of IRF5, CD226, STAT4 and IRAK1. rs4728142 is located in the promoter region of IRF 5 and rs 10488631 in the downstream region of
IRF5. (IRF5) Interferon regulatory factor 5, (CD226) DNAX accessory molecule 1, (STAT4) signal transducer and activator of transcription 4, (IRAK1) interleukin-1 receptor-associated kinase-1 
Table 1 Patient characteristics

\begin{tabular}{lc} 
Age (range) & $52.8(18.5-93.4)$ \\
Gender (female \%) & $495(80.9)$ \\
Smoking* (never \%) & $132(34.9)$ \\
Lung function follow-up length (years) & $8.2(0.1-31.8)$ \\
Presence of ILD (\%) & $394(64.4)$ \\
Baseline pulmonary function* & \\
DLCO\% predicted & $60.9(47.9-74.4)$ \\
FEV1\% predicted & $84.7(71.9-96.0)$ \\
FVC\% predicted & $88.7(73.9-104.3)$ \\
CPI & $33.4(22.9-45.9)$ \\
Mortality* (deaths \%) & $250(45.2)$ \\
Autoantibody* $\%)$ & \\
ATA & $150(26.5)$ \\
ACA & $126(22.3)$ \\
\hline
\end{tabular}

Number of patients is equal to 612 unless otherwise stated. Age is age at 1 st pulmonary function test $/ 1$ st hospital visit. Data are presented as age, mean (range), all other data are presented as median (interquartile range), except for follow-up length which is presented as median (range)

*smoking status available for $n=378$, baseline pulmonary function available for $n=583$, mortality available for $n=553$ and autoantibody status available for $n=566$

$I L D$ interstitial lung disease, $D L C O$ diffusing capacity of the lung for carbon monoxide, FEVI forced expiratory volume in $1 \mathrm{~s}, F V C$ forced vital capacity, $C P I$ composite physiological index, ATA antitopoisomerase antibody, $A C A$ anti-centromere antibody

rs 10488631 and time to decline in FVC by $\geq 10 \%$ (OR 1.42 (95\% CI 1.08-1.87), $p=0.012$ ) and with time to decline in DLCO by $\geq 15 \%$ (OR 1.32 (95\% CI 1.02-1.71), $p=0.038$ ), although neither remained significant when Bonferroni correction was applied $\left(p^{\text {corr }}=0.084\right.$ and $p^{\text {corr }}=0.27$ respectively). Both of these associations were present on multivariate analysis correcting for age at baseline, gender, smoking status, and disease severity (CPI) (FVC decline by $\geq 10 \%$ OR 1.34 (95\% CI $1.02-1.85$ ), $p=0.04$ and DLCO decline by $\geq 15 \%$ OR 1.32 (95\% CI 1.01-1.74), $p=0.044)$, although again neither remained significant following Bonferroni correction ( $p$ ${ }^{\text {corr }}=0.28$ and $p^{\text {corr }}=0.31$, respectively) (Table 3 ).

\section{Discussion}

A number of genetic associations with SSc-ILD have been reported. However, conflicting evidence exists, with only a few associations replicated in more than one study [9]. We selected seven SNPs over four genes, for which the most robust evidence of an association with SSc-ILD had been reported, with the aim to test these associations in our UK-based SSc cohort of patients of European descent.

In this study, three of the tested SNPs were significantly associated with SSc as a whole, confirming previous findings [9]. However, we found no evidence that any of the seven
SNPs are associated specifically with the presence of ILD. By contrast, we report the novel finding that the STAT4 rs7574865 Tallele may be protective against the development of lung fibrosis in SSc patients.

Although well replicated associations between the IRF5 and STAT4 SNPs are reported with SSc as a whole [4-6], conflicting results exist for genetic associations specifically with SSc-ILD. A meta-analysis of five European populations found all three IRF5 SNPs to be associated with all of the tested SSc subtypes, including, as we found in our study, no ILD [20], suggesting that the IRF5 association is with $\mathrm{SSc}$ as a whole, rather than specifically with ILD. Similarly, although the STAT4 SNP rs7574865 association with SSc-ILD has been reported in both a French [13] and a Han Chinese [7] population, a study of six European populations found no significant association in any of the populations individually, nor in the meta-analysis [21]. In fact, rs7574865 was associated with limited but not diffuse cutaneous skin disease, the phenotype more frequently associated with ILD [21]. Although a meta-analysis of three European populations found CD226 SNP rs763361 to be associated with SSc-ILD [14]; a larger study, comprising patients from seven European cohorts, did not confirm an association with the individual SNP, while reporting an association with a haplotype [22]. The IRAK1 SNP rs1059702, which we did not find to be associated with SSc-ILD nor with SSc as a whole, has been found to be associated with SSc-ILD in two meta-analysis studies of multiple European populations, although not in the individual populations $[15,16]$.

The observation that the STAT4 variant is significantly less frequent in patients with SSc-ILD compared with SSc patients without ILD is interesting. However, the findings of this study will need to be replicated in independent populations. No individual SNP is currently sufficiently strongly associated with either SSc or SSc-ILD for use in clinical diagnostics. It is possible that in the future, a panel of genetic variations, possibly combined with other biomarkers, could be utilised in the clinic to aid diagnostics or prognostics, but currently no test is sufficiently powered to provide information for an individual patient.

The current study has some limitations. Even though our SScILD cohort was fairly large for a relatively infrequent entity, being favourably comparable with published single cohort studies reporting an association with SSc-ILD [7, 10, 12], it may have been underpowered to detect small genetic effects. This is particularly true for the IRAKI SNP, a gene found on the $\mathrm{X}$ chromosome, such that only female patients $(n=483)$ and controls $(n=263)$ were included in this analysis. With these sample sizes, the study had $80 \%$ power to detect an association with an OR of at least 1.97 at $p^{\text {corr }}<0.05$, for the IRAK1 SNP, which has a minor allele frequency of 0.16 . SSc-ILD is a complex disease, and it is expected that there will be a number of genetic susceptibility loci, each with modest effect, contributing to increasing the risk of lung fibrosis. We may therefore have been unable to detect associations of small effect size and acknowledge that larger patient sample sizes derived from multicentre 
collaborations are required to definitively characterise the genetic risk of SSc-ILD. Another limitation is the lack of matching between the patient and the control population. While all patients and controls included in the study were of European descent, we were unable to account for fine-scale population structure as we did not have genome-wide data. Information on age and smoking history of the control population was not available. Although we do not expect age to influence any of the described genetic associations, smoking history could affect our interpretation as we could detect SNP associations with differences in smoking behaviours between cases and controls. As we did not have smoking information for our control population, we have performed a GWAS Catalogue search and have verified that none of the SNPs included in this study are associated with smoking behaviour.

The biological pathways involved in the susceptibility to ILD in patients with SSc remain largely unknown. Immunosuppression has been associated with modest improvement in SSc-ILD [23, 24], suggesting that immune mediated pathways are key drivers of lung fibrosis in this disease, and nintedanib, a multityrosine kinase inhibitor, has recently been approved as treatment for SSc-ILD based upon reduction in decline in lung function in the large SENSCIS clinical trial [25]. The genes investigated in this study are involved in the functioning of the immune system, with most having also been associated with other autoimmune diseases. Whether genes encoding for immune pathways are involved in the genetic predisposition to SSc-ILD uniquely, rather than with SSc as a whole, remains to be determined. On the other hand, the genetic risk may lie within genes involved in aberrant wound healing/ pro-fibrotic pathways. A progressive fibrotic phenotype despite immunosuppression is observed in a subset of patients with SSc-ILD, and anti-fibrotic treatments currently used in IPF, have recently shown promise also in SSc-ILD [25]. However, despite some similarities with IPF and other idiopathic interstitial pneumonias (IIPs), SSc-ILD appears to be genetically distinct. Loci associated with IIPs tend to be involved with hostdefence, epithelial injury/dysfunction and wound healing [26]. The gain-of-function mucin 5B (MUC5B) promoter variant, the most consistent common genetic risk factor for IIPs, is not associated with SSc-ILD [27]. Similarly, other genetic susceptibility loci identified in recent genome-wide association studies in IIPs have not been confirmed in SSc-ILD cohorts [26]. By contrast, IIP associated variants have also been found in rheumatoid arthritis-ILD, and for the MUC5B variant at least, specifically with an underlying usual interstitial pneumonia (UIP) pattern [28]. As SSc-ILD is most frequently characterised by a fibrotic non-specific interstitial pneumonia (NSIP) pattern, one can speculate that the genetic architecture may differ between these two histological patterns. However, there may instead be disease-specific genetic risks for SSc-ILD, which will require an approach focused on the development and progression of ILD in SSc patients. 
Table 3 Relationship between individual SNPs, ILD progression and survival

\begin{tabular}{|c|c|c|c|c|c|c|}
\hline \multirow[b]{2}{*}{ SNP } & \multicolumn{3}{|l|}{ Univariate } & \multicolumn{3}{|l|}{ Multivariate* } \\
\hline & Hazards ratio $(\mathrm{CI})$ & $p$ value & $p^{\text {corr }}$ value & Hazards ratio $(\mathrm{CI})$ & $p$ value & $p^{\text {corr }}$ value \\
\hline \multicolumn{7}{|l|}{ Decline in FVC $\geq 10 \%$} \\
\hline IRF5 rs4728142(G>A) & $1.23(0.86-1.77)$ & 0.25 & \multirow{7}{*}{0.084} & $1.18(0.81-1.72)$ & 0.38 & \multirow{7}{*}{0.28} \\
\hline$I R F 5$ rs2004640 (G>T) & $0.92(0.67-1.25)$ & 0.59 & & $0.95(0.68-1.32)$ & 0.75 & \\
\hline IRF5 rs10954213 (G>A) & $1.25(0.93-1.69)$ & 0.15 & & $1.16(0.84-1.61)$ & 0.35 & \\
\hline IRF5 rs10488631 (T>C) & $1.42(1.08-1.87)$ & 0.012 & & $1.34(1.02-1.85)$ & 0.04 & \\
\hline$C D 226$ rs763361 $(\mathrm{C}>\mathrm{T})$ & $1.46(0.99-2.15)$ & 0.056 & & $1.40(0.94-2.10)$ & 0.10 & \\
\hline STAT4 rs7574865 (G>T) & $0.98(0.73-1.33)$ & 0.91 & & $1.10(0.80-1.52)$ & 0.56 & \\
\hline IRAK1 rs1059702 (G>A) & $1.11(0.79-1.56)$ & 0.55 & & $1.01(0.71-1.45)$ & 0.94 & \\
\hline \multicolumn{7}{|l|}{ Decline in DLCO $\geq 15 \%$} \\
\hline IRF5 rs4728142 (G>A) & $1.39(1.00-1.92)$ & 0.05 & \multirow{7}{*}{0.27} & $1.30(0.93-1.82)$ & 0.13 & \multirow{7}{*}{0.31} \\
\hline IRF5 rs2004640 (G>T) & $0.96(0.73-1.28)$ & 0.80 & & $0.94(0.70-1.26)$ & 0.66 & \\
\hline$I R F 5$ rs10954213 (G>A) & $1.49(0.88-1.52)$ & 0.30 & & $1.15(0.86-1.52)$ & 0.35 & \\
\hline IRF5 rs10488631 (T>C) & $1.32(1.02-1.71)$ & 0.038 & & $1.32(1.01-1.74)$ & 0.044 & \\
\hline$C D 226$ rs763361 $(\mathrm{C}>\mathrm{T})$ & $1.24(0.89-1.73)$ & 0.20 & & $1.22(0.87-1.71)$ & 0.26 & \\
\hline STAT4 rs7574865 $(\mathrm{G}>\mathrm{T})$ & $0.93(0.71-1.22)$ & 0.60 & & $1.02(0.77-1.37)$ & 0.87 & \\
\hline$I R A K 1 \mathrm{rs} 1059702(\mathrm{G}>\mathrm{A})$ & $1.02(0.75-1.38)$ & 0.91 & & $1.05(0.77-1.44)$ & 0.76 & \\
\hline \multicolumn{7}{|l|}{ Mortality } \\
\hline IRF5 rs4728142 (G>A) & $0.89(0.67-1.17)$ & 0.40 & & $0.82(0.59-1.15)$ & 0.25 & \\
\hline IRF5 rs2004640 (G>T) & $0.96(0.74-1.24)$ & 0.73 & & $1.06(0.77-1.46)$ & 0.70 & \\
\hline IRF5 $\mathrm{rs} 10954213(\mathrm{G}>\mathrm{A})$ & $1.08(0.83-0.39)$ & 0.57 & & $1.03(0.75-0.39)$ & 0.87 & \\
\hline IRF5 rs10488631 (T>C) & $0.95(0.71-1.26)$ & 0.72 & & $0.77(0.54-1.11)$ & 0.16 & \\
\hline$C D 226$ rs763361 $(\mathrm{C}>\mathrm{T})$ & $0.94(0.70-1.27)$ & 0.70 & & $1.01(0.70-1.44)$ & 0.98 & \\
\hline STAT4 rs7574865 (G>T) & $0.86(0.67-1.10)$ & 0.22 & & $1.02(0.75-1.38)$ & 0.90 & \\
\hline IRAK1 rs1059702 (G>A) & $0.90(0.67-1.21)$ & 0.50 & & $1.09(0.76-1.55)$ & 0.64 & \\
\hline
\end{tabular}

*Multivariate analysis correcting for age, gender, smoking history and disease severity (CPI). $p^{\text {corr }}$ value is Bonferroni corrected for testing $7 \mathrm{SNPs}$ SNP single nucleotide polymorphism, $C I$ confidence interval, $F V C$ forced vital capacity, DLCO diffusing capacity of the lung for carbon monoxide, IRF5 interferon regulatory factor 5, CD226 DNAX accessory molecule 1, STAT4 signal transducer and activator of transcription 4, IRAK1 interleukin-1 receptor-associated kinase-1

The majority of previous studies have focused on SSc as a whole, with SSc-ILD investigated as a post hoc sub-analysis. These studies are therefore often underpowered to detect associations with SSc-ILD specifically. Furthermore, few have sought out to detect genetic risk factors for significant ILD outcomes, including lung function decline. With the availability of large cohorts with adequate longterm lung function follow-up, it should be possible to detect specific genetic associations with a progressive fibrotic phenotype and/or potential gene variants associated with increased likelihood of response to anti-inflammatory or anti-fibrotic agents. This study highlights the need for more, adequately powered, studies addressing the specific question of the genetic susceptibility to SSc-ILD. This will require international collaborations aimed at performing hypothesis-free genome-wide association studies specifically targeted at well-defined SSc-ILD cross-sectionally and longitudinally.
Funding information This work was supported by Versus Arthritis (grant no. 20719). T.M. Maher is supported by an NIHR Clinician Scientist Fellowship (NIHR Ref: CS-2013-13-017) and a British Lung Foundation Chair in Respiratory Research (C17-3). P.L. Molyneaux is supported by an Action for Pulmonary Fibrosis Mike Bray fellowship. L.V. Wain is supported by a GSK/British Lung Foundation Chair in Respiratory Research (C17-1). The research was partially supported by the National Institute for Health Research (NIHR) Leicester Biomedical Research Centre; the views expressed are those of the author(s) and not necessarily those of the National Health Service (NHS), the NIHR or the Department of Health.

\section{Compliance with ethical standards}

\section{Disclosures None.}

Ethical standards All participants gave written informed consent, and the Ethics Committees of the Royal Brompton Hospital and of the Royal Free Hospital gave authorisation for the study (REC 13/LO/0857).

Open Access This article is licensed under a Creative Commons Attribution 4.0 International License, which permits use, sharing, 
adaptation, distribution and reproduction in any medium or format, as long as you give appropriate credit to the original author(s) and the source, provide a link to the Creative Commons licence, and indicate if changes were made. The images or other third party material in this article are included in the article's Creative Commons licence, unless indicated otherwise in a credit line to the material. If material is not included in the article's Creative Commons licence and your intended use is not permitted by statutory regulation or exceeds the permitted use, you will need to obtain permission directly from the copyright holder. To view a copy of this licence, visit http://creativecommons.org/licenses/by/4.0/.

\section{References}

1. Denton CP, Khanna D (2017) Systemic sclerosis. Lancet 390: 1685-1699

2. Steen VD and Medsger, TA. Changes in causes of death in systemic sclerosis, 1972-2002.Ann Rheum Dis2007;66:940-944

3. Reveille JD, Fischbach M, McNearney T et al (2001) Systemic sclerosis in 3 US ethnic groups: a comparison of clinical, sociodemographic, serologic, and immunogenetic determinants. SeminArthritis Rheum 30:332-346

4. Sharif R, Mayes MD, Tan FK et al (2012) IRF5 polymorphism predicts prognosis in patients with systemic sclerosis. Ann.Rheum.Dis 71:1197-1202

5. Ito I, Kawaguchi Y, Kawasaki A et al (2009) Association of a functional polymorphism in the IRF5 region with systemic sclerosis in a Japanese population. Arthritis Rheum 60:1845-1850

6. Xu Y, Wang W, Tian Y, Liu J, Yang R (2016) Polymorphisms in STAT4 and IRF5 increase the risk of systemic sclerosis: a metaanalysis. Int.J.Dermatol. 55:408-416

7. Yi L, Wang JC, Guo XJ et al (2013) STAT4 is a genetic risk factor for systemic sclerosis in a Chinese population. Int.J.Immunopathol.Pharmacol 26:473-478

8. Radstake TR, Gorlova O, Rueda B, Martin JE, Alizadeh BZ, Palomino-Morales R, Coenen MJ, Vonk MC, Voskuyl AE, Schuerwegh AJ, Broen JC, van Riel P, van 't Slot R, Italiaander A, Ophoff RA, Riemekasten G, Hunzelmann N, Simeon CP, Ortego-Centeno N, González-Gay MA, González-Escribano MF, Spanish Scleroderma Group, Airo P, van Laar J, Herrick A, Worthington J, Hesselstrand R, Smith V, de Keyser F, Houssiau F, Chee MM, Madhok R, Shiels P, Westhovens R, Kreuter A, Kiener H, de Baere E, Witte T, Padykov L, Klareskog L, Beretta L, Scorza R, Lie BA, Hoffmann-Vold AM, Carreira P, Varga J, Hinchcliff M, Gregersen PK, Lee AT, Ying J, Han Y, Weng SF, Amos CI, Wigley FM, Hummers L, Nelson JL, Agarwal SK, Assassi S, Gourh P, Tan FK, Koeleman BP, Arnett FC, Martin J, Mayes MD (2010) Genome-wide association study of systemic sclerosis identifies CD247 as a new susceptibility locus. Nat.Genet. 42:426-429

9. Stock CJW, Renzoni EA (2018) Genetic predictors of systemic sclerosis-associated interstitial lung disease: a review of recent literature. Eur.J.Hum. Genet 26:765-777

10. Dieude P, Guedj M, Wipff J et al (2009) Association between the IRF5 rs2004640 functional polymorphism and systemic sclerosis: a new perspective for pulmonary fibrosis. Arthritis Rheum 60:225-233

11. Dieude P, Dawidowicz K, Guedj M, Legrain Y, Wipff J, Hachulla E, Diot E, Sibilia J, Mouthon L, Cabane J, Amoura Z, Crakowski JL, Carpentier P, Avouac J, Meyer O, Kahan A, Boileau C, Allanore Y (2010) Phenotype-haplotype correlation of IRF5 in systemic sclerosis: role of 2 haplotypes in disease severity. J.Rheumatol. 37:987-992

12. Wang J, Yi L, Guo X, Liu M, Li H, Zou H, Gu Y, Tu W, Guo G, Yang L, Lai S, He D, Zhou X (2014) Association of the IRF5 SNP rs2004640 with systemic sclerosis in Han Chinese. Int.J.Immunopathol.Pharmacol. 27:635-638

13. Dieude P, Guedj M, Wipff J et al (2009) STAT4 is a genetic risk factor for systemic sclerosis having additive effects with IRF5 on disease susceptibility and related pulmonary fibrosis. Arthritis Rheum 60:2472-2479

14. Dieude P, Guedj, M., Truchetet, ME. et al.Association of the CD226 Ser(307) variant with systemic sclerosis: evidence of a contribution of costimulation pathways in systemic sclerosis pathogenesis. Arthritis Rheum2011;63:1097-1105

15. Dieude P, Bouaziz M, Guedj M et al (2011) Evidence of the contribution of the $\mathrm{X}$ chromosome to systemic sclerosis susceptibility: association with the functional IRAK1 196Phe/532Ser haplotype. Arthritis Rheum 63:3979-3987

16. Carmona FD, Cenit MC, Diaz-Gallo LM et al (2013) New insight on the Xq28 association with systemic sclerosis. Ann.Rheum.Dis

17. (1980) Preliminary criteria for the classification of systemic sclerosis (scleroderma). Subcommittee for scleroderma criteria of the American Rheumatism Association Diagnostic and Therapeutic Criteria Committee. Arthritis Rheum 23:581-590

18. Auton A, Brooks LD, Durbin RM et al (2015) A global reference for human genetic variation. Nature 526:68-74

19. Wells AU, Desai, SR, Rubens, M. B. et al. Idiopathic pulmonary fibrosis: a composite physiologic index derived from disease extent observed by computed tomography. Am J Respir Crit Care Med2003;167:962-969

20. Carmona FD, Martin JE, Beretta L et al (2013) The systemic lupus erythematosus IRF5 risk haplotype is associated with systemic sclerosis. PLoS.One 8:e54419

21. Rueda B, Broen J, Simeon C, Hesselstrand R, Diaz B, Suárez H, Ortego-Centeno N, Riemekasten G, Fonollosa V, Vonk MC, van den Hoogen F, Sanchez-Román J, Aguirre-Zamorano MA, GarcíaPortales R, Pros A, Camps MT, Gonzalez-Gay MA, Coenen MJ, Airo P, Beretta L, Scorza R, van Laar J, Gonzalez-Escribano MF, Nelson JL, Radstake TR, Martin J (2009) The STAT4 gene influences the genetic predisposition to systemic sclerosis phenotype. Hum.Mol.Genet. 18:2071-2077

22. Bossini-Castillo L, Simeon CP, Beretta L et al (2012) A multicenter study confirms CD226 gene association with systemic sclerosisrelated pulmonary fibrosis. Arthritis Res.Ther 14:R85

23. Tashkin DP, Elashoff R, Clements PJ et al (2006) Cyclophosphamide versus placebo in scleroderma lung disease. N.Engl.J.Med 354:2655-2666

24. Tashkin DP, Roth MD, Clements PJ et al (2016) Mycophenolate mofetil versus oral cyclophosphamide in scleroderma-related interstitial lung disease (SLS II): a randomised controlled, double-blind, parallel group trial. Lancet Respir.Med 4:708-719

25. Distler O, Highland KB, Gahlemann M et al (2019) Nintedanib for systemic sclerosis-associated interstitial lung disease. N.Engl.J.Med 380:2518-2528

26. Wu M, Assassi S, Salazar GA et al (2016) Genetic susceptibility loci of idiopathic interstitial pneumonia do not represent risk for systemic sclerosis: a case control study in Caucasian patients. Arthritis Res.Ther 18:20

27. Stock CJ, Sato H, Fonseca C et al (2013) Mucin 5B promoter polymorphism is associated with idiopathic pulmonary fibrosis but not with development of lung fibrosis in systemic sclerosis or sarcoidosis. Thorax 68:436-441

28. Juge PA, Lee JS, Ebstein E et al (2018) MUC5B promoter variant and rheumatoid arthritis with interstitial lung disease. N.Engl.J.Med 379:2209-2219

Publisher's note Springer Nature remains neutral with regard to jurisdictional claims in published maps and institutional affiliations. 